\title{
Pre- and Postoperative Parameters on Magnetic Resonance Imaging Predict Continence Recovery after Laparoscopic Radical prostatectomy
}

\section{Fan Zhang}

Peking University Third Hospital

Bin Yang

Peking University Third Hospital

Ye Yan

Peking University Third Hospital

Yichang Hao

Peking University Third Hospital

Yi Huang

Peking University Third Hospital

Lulin Ma ( $\nabla$ malulin@bjmu.edu.cn )

Peking University Third Hospital

\section{Research article}

Keywords: Prostatectomy, Continence, Magnetic resonance imaging, Parameters

Posted Date: July 17th, 2020

DOl: https://doi.org/10.21203/rs.3.rs-31551/v1

License: () (1) This work is licensed under a Creative Commons Attribution 4.0 International License. Read Full License 


\section{Abstract}

Background: To evaluate the association between pre- and postoperative parameters on magnetic resonance imaging (MRI) and continence recovery after laparoscopic radical prostatectomy (LRP). To develop a risk scoring system for predicting continence recovery after LRP.

Methods: 73 patients who underwent LRP were retrospectively reviewed. Demographic characteristics, clinicopathologic outcomes and several MRI parameters before and after surgery were evaluated. Continence was defined as no pad per day. Early continence recovery was defined as continence recovery within 3 months. Kaplan-Meier analyses and log-rank test were used to compare time to continence recovery. Cox proportional-hazards regression analyses were performed to identify independent predictors of continence recovery.

Results: Patients with smaller prostatic volume, shorter intravesical prostatic protrusion length (IPPL), shorter preoperative membranous urethral length (MUL), lower MUL-removal rate, triangular vesicourethral anastomosis (VUA) and neurovascular bundle sparing experienced a faster continence recovery (All, $p<0.05$ ). Multivariate analyses revealed IPPL (hazard ratio [HR]: 0.94, $p=0.044$ ), preoperative MUL (HR: 1.10, $p=0.032)$, MUL-removal rate (HR: 0.91, $p=0.007$ ) and shape of VUA (square vs. triangle, HR: 2.30, $p=0.012$ ) were independent predictors of continence recovery. The four parameters were therefore used to develop a risk scoring system, termed Post-Prostatectomy Incontinence Score (PPIS) and ranging from 0 to 4 . We observed early continence recovery in $100 \%, 84.6 \%, 27.8 \%, 0 \%$ and $0 \%$ of patients with a PPIS of $0,1,2,3,4$, respectively.

Conclusion: IPPL, preoperative MUL, MUL-removal rate and shape of VUA were independently predictors of continence recovery. PPIS could accurately predict the early continence recovery after LRP.

\section{Background}

Radical prostatectomy (RP) is the main treatment option for localized prostate cancer, which obtains favorable oncological outcomes [1]. However, post-prostatectomy incontinence (PPI) is a bothersome complication with a significant negative effect on the health-related quality of life [2]. A systemic review showed that $4-31 \%$ of patients complain of urinary incontinence in 12 months after RP, with a "No Pad" definition of PPI [3].

Despite the etiology of PPI is multifactorial and complex, reduction of internal urethral pressure is the main factor. Several clinical parameters have been identified to be predictors of PPI, including age, body mass index (BMI), metabolic syndrome, prostate volume (PV), preoperative overactive bladder and neurovascular bundle (NVB) sparing [4-6]. Furthermore, urologists have proposed to predict continence recovery with preoperative anatomical parameters on magnetic resonance imaging (MRI) such as MUL and intravesical prostatic protrusion length (IPPL) $[7,8]$. However, the role of postoperative anatomical parameters in continence recovery has not been evaluated sufficiently. For better patient counseling and early intervention, it is important to identified the risk factors for PPI. The objective of the study was to 
evaluate the association between pre- and postoperative anatomical parameters on MRI and continence recovery after laparoscopic radical prostatectomy (LRP), and to develop a risk scoring system to predict the early continence recovery.

\section{Methods}

\section{Study population}

The retrospective study was conducted in 74 patients treated with LRP for localized prostate cancer at our institute from January 2015 to April 2019 and was approved by the Institutional Review Board of Peking University Third Hospital. All patients underwent pre- and postoperative MRI to evaluate the pelvic anatomical parameters. None of the patients had underwent transurethral resection or holmium laser enucleation of the prostate. No patients had history of incontinence or urethral stricture. No patients received neoadjuvant hormone therapy or radiotherapy. The clinical and pathological data was reviewed in our collected database.

\section{Surgical procedures}

All patients received extraperitoneal LRP. After cutting part of the puboprostatic ligament, the deep dorsal vein was suture-ligated. NVB-sparing procedure was attempted to perform for all clinical localized prostate cancer. Reconstruction was performed using full-thickness interrupted sutures when needed. Urethro-vesical anastomosis was performed with 3-0 V-Loc sutures continuously.

\section{MRI parameters measurement}

MRI was performed on a 3.0 Tesla magnetic resonance scanner. The mean interval from LRP to postoperative MRI was $12.9 \pm 7.0$ months. Parameters measurement was performed on T2-weighted MRI images. PV was calculated by the formula, i.e. PV $=0.52 *$ length* width*height. IPPL was measured as the vertical distance from the tip of the protruding prostate to the base of the urinary bladder on the midsagittal image (Fig 1).. Preoperative MUL was defined as the distance from the apex of prostate to the level of the urethra at penile bulb on the coronal image (Fig 2a).. Postoperative MUL was defined as the distance from the bladder neck to the level of the urethra at the penile bulb on the coronal image (Fig 2 b).. MUL-removal rate was calculated by the formula, i.e. MUL-removal rate $=($ Preoperative MUL Postoperative MUL) / Preoperative MUL. The shape of vesicourethral anastomosis (VUA) on the midsagittal image was classified into triangle and square (Fig 3a and 3b).. PV, IPPL, pre- and postoperative MUL, and shape of VUA were evaluated by one observer who were blinded to the clinical results.

\section{Continence evaluation}


We routinely instructed the patients of pelvic floor muscle exercises to acquire the continence recovery after surgery. They were followed up by outpatient service or telephone interviews every 3 months. In the study, urinary continence was defined as freedom from using safety pad ( 0 pad/day). Early continence recovery was defined as continence recovery within 3 months.

\section{Statistical analyses}

All statistical analyses were conducted with SPSS statistics version 22.0 (IBM Corp, Armonk, NY, USA). Continuous variables were presented as the mean value and standard deviation. The comparison between discontinuous variables and continuous variables was evaluated by Chi-square test and Student's t test, respectively. Kaplan-Meier analyses and log-rank test were used to compare time to continence recovery between groups. Univariate and multivariate Cox proportional-hazards regression analyses were used to identify independent predictors of continence recovery after LRP. A risk scoring system based on the independent predictors was developed to classified patients according to the likelihood of the continence recovery, and the area under the receiver operating characteristic curve (AUC) on continence recovery was evaluated. All tests of significance were two sided, and $p<0.05$ was considered statistical significance.

\section{Results}

The clinical and pathological characteristics of patients are summarized in Table 1. The mean age was $69.8 \pm 7.7$ years. Patients with an mean BMl of $25.0 \pm 3.2 \mathrm{~kg} / \mathrm{m}^{2}$. The mean operation time was $227 \pm 68$ min, and the mean estimated blood loss was $106 \pm 123 \mathrm{ml}$. Non-, unilateral, and bilateral NVB sparing LRP was performed in $22(30.1 \%), 4(5.5 \%)$ and $47(64.4 \%)$ patients, respectively. There were 5(6.8\%), $27(40.0 \%)$ and $41(56.2 \%)$ patients with Gleason score 6,7 and $\geq 8$, respectively. 32(43.8\%) patients suffered a positive surgical margin. The mean PV was $39.6 \pm 22.3 \mathrm{ml}$, the mean IPPL was $2.8 \pm 4.6 \mathrm{~mm}$, the mean preoperative MUL was $13.1 \pm 3.2 \mathrm{~mm}$ and the mean MUL-removal rate was $6.0 \%$. There were $50(68.5 \%)$ patients with triangle VUA, $23(31.5 \%)$ patients with square VUA on the postoperative MRI.

In the study $57.5 \%(42 / 73)$ of patients achieved early continence recovery after LRP. Continence recovery was achieved in $78.1 \%(57 / 73)$ and $97.3 \%$ (71/73) of patients within 6 , and 12 months after LRP, respectively. Patients achieving early continence recovery had significant shorter operation time (210 min vs. $249 \mathrm{~min}, p=0.024)$, less estimated blood loss ( $75 \mathrm{ml}$ vs. $148 \mathrm{ml}, p=0.029)$, smaller PV (33.7 ml vs. $47.5 \mathrm{ml}, p=0.008)$, shorter IPPL ( $0.9 \mathrm{~mm}$ vs. $5.4 \mathrm{~mm}, p<0.001)$, longer preoperative MUL ( $14.3 \mathrm{~mm}$ vs. $11.6 \mathrm{~mm}, p<0.001)$, lower MUL-removal rate (1.9\% vs. $11.7 \%, p<0.001)$, higher prevalence of triangle VUA $(p<0.001)$ and NVB sparing $(p=0.014)$ (Table 1).. There was no significant difference between the two groups in age, BMI, PSA, and pathological characteristics such as Gleason score, pathological T stage and positive surgical margin (Table 1).. 
Kaplan-Meier analyses and log-rank test revealed that NVB sparing status (yes vs. no, $p=0.005$ ), PV (<40ml vs. $\geq 40 \mathrm{ml}, p=0.001)$, IPPL ( $\nabla 5 \mathrm{~mm}$ vs. $\geq 5 \mathrm{~mm}, p=0.001)$, preoperative MUL ( $\geq 15 \mathrm{~mm}$ vs. $<15 \mathrm{~mm}, p<0.001)$, MUL-removal rate (<10\% vs. $\geq 10 \%, p<0.001)$ and shape of VUA (square vs. triangle, $p<0.001$ ) were all significantly associated with continence recovery (Supplemental Fig 1).. Univariate Cox proportional analyses revealed NVB sparing status $(p=0.021)$, $\mathrm{PV}(p=0.016), \operatorname{IPPL}(p=0.002)$, preoperative MUL $(p=0.004)$, MUL-removal rate $(p<0.001)$ and shape of VUA $(p<0.001)$ were predictors of continence recovery after LRP (Table 2).. On the multivariate analyses, only IPPL (hazard ratio [HR]: 0.94, 95\% confidence interval [Cl]: 0.88-1.00, $p=0.044)$, preoperative MUL (HR: 1.10, 95\% Cl: 1.01-1.19, $p=0.032$ ), MUL-removal rate (HR: $0.91,95 \% \mathrm{Cl}: 0.86-0.98, p=0.007$ ) and shape of VUA (square vs. triangle, HR: $2.30,95 \% \mathrm{Cl}: 1.21-4.39, p=0.012)$ were identified to be independent predictors of continence recovery after LRP (Table 2)..

IPPL ( $₫ 5 \mathrm{~mm}=0$ score, $\geq 5 \mathrm{~mm}=1$ score), preoperative MUL ( $\geq 15 \mathrm{~mm}=0$ score,$<15 \mathrm{~mm}=1 \mathrm{score})$, MULremoval rate $(\nabla 10 \%=0$ score,$\geq 10 \%=1$ score) and shape of VUA (triangle $=0$ score, square $=1$ score) were then summed to develop a risk scoring system, termed Post-Prostatectomy Incontinence Score (PPIS) that ranges from 0 to 4 (Table 3).. We observed early continence recovery in $100 \%, 84.6 \%, 27.8 \%$, $0 \%$ and $0 \%$ of patients with a PPIS of $0,1,2,3,4$, respectively. The PPIS achieved a satisfactory AUC of 0.93 (95\% Cl: 0.87-0.99) for predicting early continence recovery after LRP (Fig 4), AUCs of 0.89 (95\% Cl: $0.80-0.97)$ and 0.92 (95\% Cl: 0.83-1.00) for predicting continence recovery within 6 and 12 months, respectively.

\section{Discussion}

Incontinence remains to be one of the most significant factors affecting quality of life after RP. In the present study, $57.5 \%$ of patients achieved early continence recovery, which was consistent with previous studies $[4,9]$. With the advances in knowledge of urinary continence mechanism, the main structure in continence is considered the external striated sphincter, which maintains a urethral closure pressure greater than bladder pressure. Sphincteric incompetence after RP may be a result of shorter MUL, loss of neural innervations, muscle damage and loss of the surrounding support tissue [10]. Though PPI is usually a temporary symptom, the lack of information predicting continence recovery leads to anxiety in patients and prevents early treatment for PPI. Therefore, to identify possible predictors of PPI and develop a risk scoring system can contribute to patient counseling and early intervention of PPI.

Advanced age has been proposed as an important predictor of continence recovery after surgery by some studies $[4,11,12]$. In contrast, our study revealed that there was not significant difference on ages between patients with and without early continence recovery. NyarangiDix et al [13] suggested that continence recovery in elderly men does not differ from younger men undergoing robot-assisted radical prostatectomy. Patient characteristics, surgeon experience and surgical techniques play an important role in continence recovery after RP. NVB sparing was confirmed to be an effective surgical technique for improving continence recovery $[14,15]$. Our study also revealed that patients treated with NVB sparing achieved significant better continence recovery. 
We identified that preoperative MUL was an independent predictor of continence recovery. Coakley et al [16] firstly revealed that longer preoperative MUL was associated with faster continence recovery. The study reported $89 \%$ of patients with a MUL > $12 \mathrm{~mm}$ achieved continence recovery compared with $77 \%$ of patients with a preoperative MUL $\leq 12 \mathrm{~mm}$ in 12 months after surgery. A meta-analysis containing one randomized controlled trial and 12 cohort studies demonstrated that a longer preoperative MUL is significantly and positively associated with continence recovery after RP [17]. Matsushita et al [18] reported that the addition of preoperative MUL increased the AUC of model for predicting continence recovery, but the AUC was not good enough. Longer MUL including a greater amount of external striated sphincter, play an important role in maintaining and increasing the urethral closure pressures.

RP leads to damages in the structure and function of membranous urethra. However, the MUL-removal rates can vary greatly between different patients and surgeons. The postoperative MUL may be a more accurate parameter for predicting continence recovery than preoperative MUL. Since MRI is not routine performed after RP, only a few studies revealed that longer postoperative MUL was associated with a faster continence recovery after PR $[7,9,19]$. Postoperative MUL measured on cystourethrography was also identified to be an important predictor of continence recovery [20]. Kohjimoto et al [21] examined 179 prostate specimens of RP and identified that MUL removed with prostate was an independent predictor of continence recovery. Our study revealed that patients with higher MUL-removal rate suffered a significantly longer period of PPI. Therefore, blunt dissection of the urethra distal to the prostatic apex should be carried out for sparing the membranous urethra, which could contribute to improving continence recovery after RP [22].

In recent studies, several MRI parameters such as IPPL, bladder neck width were identified to be predictors of continence recovery after RP [8,9]. Lee et al. [8] reported that significant improvement in continence recovery was observed in patients with IPPL $<5 \mathrm{~mm}$ at all periods compared with those with IPPL $\geq 5 \mathrm{~mm}$. In a cohort of 821 patients who underwent robot-assisted radical prostatectomy, IPPL measured by transrectal ultrasound was also identified to be a powerful predictor of continence recovery [23]. Our previous study also confirmed that patients achieved early continence recovery after LRP had a significant shorter IPPL [24]. The core condition of urinary continence is the balance of detrusor contractility and urethral pressure. Intravesical prostatic protrusion was identified to be associated with lower urinary tract symptoms and overactive bladder $[25,26]$. It is supposed that longer IPPL contributes to pathophysiological changes of bladder detrusor such as detrusor hyperactive, detrusor instability and subsequent bladder dysfunction, and then leads to a delay in continence recovery after RP. Furthermore, the impact of intravesical prostatic protrusion on more surgical damage of the internal sphincter during bladder neck dissections put forward as a possible contributor [25]. However, the underlying mechanisms by which IPPL affects continence recovery after RP remains unclear.

On the other hand, we reveled that square UVA was a strongly negative predictor of continence recovery after RP. The risk of PPI for patients with square UVA was 2.3 times higher than those with triangle UVA. However, the mechanisms resulting in the difference on the shape of UVA is unclear. It may be related to the procedure of dissociation proximal urethra and vesical-urethral anastomosis. We proposed that 
patient with square UVA may suffered more damage such as scarring and fibrosis to the membranous urethra, which may be caused by high tension anastomosis, subsequent tissue ischemia and mechanical damage to sphincter [27]. Compared to the triangle UVA, square UVA may be an important signal of insufficient urethral closure pressure. Haga et al [19] found that patients with triangle bladder neck had better urinary continence, but it failed to showed significant difference. Further research on the association between the shape of UVA and continence recovery after RP is necessary. Finally, we develop the PPIS with the independent predictors of continence recovery after LRP in our study. It presented a satisfied ROC for predicting continence recovery after LRP. To the best of our knowledge, PPIS is the first risk scoring system based on pre- and postoperative MRI for predicting continence recovery after LRP. And the external validation studies are needed to be carried out.

The present study has some limitations. The retrospective nature of the study and the selection of patients with postoperative MRI could have generated unanticipated biases. The postoperative MRIs were performed in different intervals after surgery, which may affect the urethral parameters. Furthermore, the present study included a relatively small number of patients. Finally, the surgeon experience was not adjusted which may affect continence recovery after RP.

\section{Conclusion}

Our study identified that IPPL, preoperative MUL, MUL-removal rate and shape of VUA were independently predictors of continence recovery after LRP. PPIS is an MRI-based risk scoring system, which could accurately predict the continence recovery after LRP. The use of PPIS can help urologists to stratify patients who underwent LRP into subgroups with different risks of PPI.

\section{Abbreviations}

MRI: magnetic resonance imaging; LRP: laparoscopic radical prostatectomy; IPPL: intravesical prostatic protrusion length; MUL: membranous urethral length; VUA: vesicourethral anastomosis; PPIS: PostProstatectomy Incontinence Score; RP: radical prostatectomy; PPI: post-prostatectomy incontinence; BMI: body mass index; PV: prostate volume; NVB: neurovascular bundle; AUC: area under the receiver operating characteristic curve.

\section{Declarations}

\section{Ethics approval and consent to participate}

This study received institutional board approval at Peking University Third Hospital Medical Science Research Ethics Committee (IRB M2020235).

\section{Consent for publication}

The manuscript is approved by all authors for publication. 
Availability of data and materials

The datasets used and/or analyzed during the current study are available from the corresponding author on reasonable request.

\section{Competing interests}

All authors declare no potential conflicts of interest.

\section{Funding}

Not applicable.

\section{Authors' Contributions}

LLM, YH (Yi Huang) and FZ proposed the protocol. BY, FZ, YCH (Yichang Hao) and YY contributed to data collection and management. BY contributed to data analysis and statistical analysis. BY and FZ contributed to manuscript writing. LLM and $\mathrm{YH}$ (Yi Huang) revised the manuscript. All authors have read and approved the manuscript.

\section{Acknowledgements}

Not applicable

\section{References}

1. Hruza M, Bermejo JL, Flinspach B, et al. Long-term oncological outcomes after laparoscopic radical prostatectomy. BJU Int. 2013;111(2):271-280.

2. Punnen $\mathrm{S}$, Cowan JE, Chan JM, et al. Long-term health-related quality of life after primary treatment for localized prostate cancer: results from the CaPSURE registry. Eur Urol. 2015;68(4):600-8.

3. Ficarra V, Novara G, Rosen RC, et al. Systematic review and meta-analysis of studies reporting urinary continence recovery after robot-assisted radical prostatectomy. Eur Urol. 2012;62(3):405-417.

4. Lee YJ, Jung JW, Lee S, et al. Contemporary trends in radical prostatectomy and predictors of recovery of urinary continence in men aged over 70 years: comparisons between cohorts aged over 70 and less than 70 years. Asian J Androl. 2020;22(3):280-6.

5. Morlacco A, Dal Moro F, Rangel LJ, et al. Impact of metabolic syndrome on functional outcomes and complications of surgical treatment of prostate cancer. J Surg Oncol. 2019;120(8):1505-7.

6. Yamada $\mathrm{Y}$, Fujimura T, Fukuhara $\mathrm{H}$, et al. Overactive bladder is a negative predictor of achieving continence after robot-assisted radical prostatectomy. Int J Urol. 2017;24(10):749-756. 
7. Paparel P, Akin O, Sandhu JS, et al. Recovery of urinary continence after radical prostatectomy: association with urethral length and urethral fibrosis measured by preoperative and postoperative endorectal magnetic resonance imaging. Eur Urol. 2009;55(3):629-637.

8. Lee $\mathrm{CH}, \mathrm{Ha} \mathrm{HK}$. Intravesical prostatic protrusion as a predictor of early urinary continence recovery after laparoscopic radical prostatectomy. Int J Urol. 2014;21(7):653-6.

9. Nakane A, Kubota $H$, Noda $Y$, et al. Improvement in early urinary continence recovery after roboticassisted radical prostatectomy based on postoperative pelvic anatomic features: a retrospective review. BMC Urol. 2019;19(1):87.

10. Heesakkers J, Farag F, Bauer RM, et al. Pathophysiology and Contributing Factors in Postprostatectomy Incontinence: A Review. Eur Urol. 2017;71(6):936-944.

11. Jiang DG, Xiao CT, Mao YH, et al. Impact and Predictive Value of Prostate Weight on the Outcomes of Nerve Sparing Laparoscopic Radical Prostatectomy in Patients with Low Risk Prostate Cancer. Urol J. 2019;16(3):260-6.

12. Lee H, Kim K, Hwang SI, et al. Impact of prostatic apical shape and protrusion on early recovery of continence after robot-assisted radical prostatectomy. Urology. 2014;84(4):844-9.

13. Nyarangi-Dix JN, Tosev G, Damgov I, et al. Recovery of pad-free continence in elderly men does not differ from younger men undergoing robot-assisted radical prostatectomy for aggressive prostate cancer. World J Urol. 2020;38(2):351-360.

14. Suardi N, Moschini M, Gallina A, et al. Nerve-sparing approach during radical prostatectomy is strongly associated with the rate of postoperative urinary continence recovery. BJU Int. 2013;111(5):717-722.

15. Reeves F, Preece P, Kapoor J, et al. Preservation of the neurovascular bundles is associated with improved time to continence after radical prostatectomy but not long-term continence rates: results of a systematic review and meta-analysis. Eur Urol. 2015;68(2):692-704.

16. Coakley FV, Eberhardt S, Kattan MW, et al. Urinary continence after radical retropubic prostatectomy: relationship with membranous urethral length on preoperative endorectal magnetic resonance imaging. J Urol. 2002;168(3):1032-5.

17. Mungovan SF, Sandhu JS, Akin O, et al. Preoperative Membranous Urethral Length Measurement and Continence Recovery Following Radical Prostatectomy: A Systematic Review and Meta-analysis. Eur Urol. 2017;71(3):368-378.

18. Matsushita K, Kent MT, Vickers AJ, et al. Preoperative predictive model of recovery of urinary continence after radical prostatectomy. BJU Int. 2015;116(4):577-583.

19. Haga N, Ogawa S, Yabe M, et al. Association between postoperative pelvic anatomic features on magnetic resonance imaging and lower tract urinary symptoms after radical prostatectomy. Urology. 2014;84(3):642-9.

20. Haga N, Ogawa S, Yabe M, et al. Factors Contributing to Early Recovery of Urinary Continence Analyzed by Pre- and Postoperative Pelvic Anatomical Features at Robot-Assisted Laparoscopic Radical Prostatectomy. J Endourol. 2015;29(6):683-690. 
21. Kohjimoto Y, Yamashita S, Kikkawa K, et al. The Association of Length of the Resected Membranous Urethra With Urinary Incontinence After Radical Prostatectomy. Urol J. 2020;17(2):146-151.

22. Sfoungaristos $S$, Kontogiannis S, Perimenis P. Early continence recovery after preservation of maximal urethral length until the level of verumontanum during radical prostatectomy: primary oncological and functional outcomes after 1 year of follow-up. Biomed Res Int. 2013;2013:426208.

23. Jo JK, Hong SK, Byun SS, et al. Urinary Continence after Robot-Assisted Laparoscopic Radical Prostatectomy: The Impact of Intravesical Prostatic Protrusion. Yonsei Med J. 2016;57(5):11451151.

24. Yang B, Zhang F, Xiao C, et al. Impact of Preoperative Magnetic Resonance Imaging Anatomic Features on Urinary Continence Recovery after Laparoscopic Radical Prostatectomy. Urol Int. 2020;104(3-4):239-246.

25. Mehraban D. Clinical value of intravesical prostatic protrusion in the evaluation and management of prostatic and other lower urinary tract diseases. Asian J Urol. 2017;4(3):174-180.

26. Kim KH, Kim YS. Correlation of male overactive bladder with intravesical prostatic protrusion. Korean J Urol. 2010;51(12):843-6.

27. Momozono H, Miyake $\mathrm{H}$, Miyazaki A, et al. Significance of urethral fibrosis evaluated by preoperative magnetic resonance imaging as a predictor of continence status after robot-assisted radical prostatectomy. Int J Med Robot. 2016;12(3):496-501.

\section{Tables}


Table 1 Clinical and pathological characteristics of total patients and groups according to the early continence recovery after laparoscopic radical prostatectomy

\begin{tabular}{|c|c|c|c|c|}
\hline \multirow[t]{2}{*}{ Variables } & \multirow{2}{*}{$\begin{array}{l}\text { Total } \\
\text { 冈n=73囚 }\end{array}$} & \multicolumn{2}{|c|}{ Early continence recovery } & \multirow[t]{2}{*}{$P$ value } \\
\hline & & Yes $(n=42)$ & No $(n=31)$ & \\
\hline Age, mean \pm SD (years) & $69.8 \pm 7.7$ & $68.8 \pm 7.8$ & $71.1 \pm 7.6$ & 0.210 \\
\hline $\mathrm{BMI}$, mean $\pm \mathrm{SD}(\mathrm{kg} / \mathrm{m} 2)$ & $25.0 \pm 3.2$ & $24.8 \pm 2.6$ & $25.2 \pm 3.9$ & 0.577 \\
\hline PSA, mean \pm SD $(\mu \mathrm{g} / \mathrm{L})$ & $25.5 \pm 41.6$ & $19.0 \pm 28.6$ & $34.3 \pm 53.8$ & 0.159 \\
\hline Operation time, mean \pm SD (min) & $227 \pm 68$ & $210 \pm 52$ & $249 \pm 81$ & 0.024 \\
\hline Estimated blood loss, mean $\pm \mathrm{SD}(\mathrm{ml})$ & $106 \pm 123$ & $75 \pm 51$ & $148 \pm 172$ & 0.029 \\
\hline Gleason score & & & & 0.406 \\
\hline 6 & $5(6.8)$ & 2 & 3 & \\
\hline 7 & $27(40.0)$ & 18 & 9 & \\
\hline$\geq 8$ & $41(56.2)$ & 22 & 19 & \\
\hline Pathological stage & & & & 0.736 \\
\hline T2 & $37(50.7)$ & 22 & 15 & \\
\hline$\geq \mathrm{T} 3$ & $36(49.3)$ & 20 & 16 & \\
\hline Positive surgical margin & & & & 0.250 \\
\hline No & $41(56.2)$ & 26 & 15 & \\
\hline Yes & $32(43.8)$ & 16 & 16 & \\
\hline NVB sparing status & & & & 0.014 \\
\hline No & $22(30.1)$ & 7 & 15 & \\
\hline Unilateral & $4(5.5)$ & 3 & 1 & \\
\hline Bilateral & $47(64.4)$ & 32 & 15 & \\
\hline $\mathrm{PV}$, mean $\pm \mathrm{SD}(\mathrm{ml})$ & $39.6 \pm 22.3$ & $33.7 \pm 17.7$ & $47.5 \pm 25.6$ & 0.008 \\
\hline IPPL, mean \pm SD $(\mathrm{mm})$ & $2.8 \pm 4.6$ & $0.9 \pm 2.1$ & $5.4 \pm 5.8$ & $<0.001$ \\
\hline preoperative MUL, mean \pm SD $(\mathrm{mm})$ & $13.1 \pm 3.2$ & $14.3 \pm 2.7$ & $11.6 \pm 3.3$ & $<0.001$ \\
\hline postoperative MUL, mean \pm SD $(\mathrm{mm})$ & $12.4 \pm 3.3$ & $14.0 \pm 2.6$ & $10.1 \pm 2.9$ & $<0.001$ \\
\hline MUL-removal rate, $(\%)$ & $6.0 \pm 6.2$ & $1.9 \pm 3.7$ & $11.7 \pm 4.0$ & $<0.001$ \\
\hline Shape of VUA & & & & $<0.001$ \\
\hline Triangle & $\begin{array}{l}50(68.5) \\
\text { Page } 11 / 15\end{array}$ & 38 & 12 & \\
\hline
\end{tabular}


SD, standard deviation; BMI, body mass index; LUST, lower urinary symptom; PSA, prostate specific antigen; PV, prostatic volume; IPPL, intravesical prostatic protrusion length; MUL, membranous urethral length; NVB, neurovascular bundle; VUA, vesicourethral anastomosis

Table 2. Univariate and multivariate analysis of parameters predicting continence recovery after laparoscopic radical prostatectomy.

\begin{tabular}{|c|c|c|c|}
\hline \multirow[t]{2}{*}{ Variables } & \multirow{2}{*}{$\begin{array}{l}\text { Univariate } \\
\mathrm{P} \text { value }\end{array}$} & \multicolumn{2}{|l|}{ Multivariate } \\
\hline & & $\mathrm{HR}(95 \% \mathrm{Cl})$ & $P$ value \\
\hline Age (years) & 0.186 & & \\
\hline BMI (kg/m2】 & 0.331 & & \\
\hline Operation time (min) & 0.229 & & \\
\hline Estimated blood loss (ml) & 0.108 & & \\
\hline NVB sparing status (Yes vs. No) & 0.021 & $0.74(0.43-1.30)$ & 0.297 \\
\hline PV (ml) & 0.016 & $1.00(0.99-1.01)$ & 0.991 \\
\hline IPPL (mm) & 0.002 & $0.94(0.88-1.00)$ & 0.044 \\
\hline Preoperative MUL (mm) & 0.004 & $1.10(1.01-1.19)$ & 0.032 \\
\hline MUL-removal rate (\%) & $<0.001$ & $0.91(0.86-0.98)$ & 0.007 \\
\hline Shape of VUA (square vs. triangle) & $<0.001$ & $2.30(1.21-4.39)$ & 0.012 \\
\hline
\end{tabular}

\begin{tabular}{|c|c|c|}
\hline PPIS & 0 score & 1 score \\
\hline Intravesical prostatic protrusion length (mm) & $85 \mathrm{~mm}$ & $\geq 5 \mathrm{~mm}$ \\
\hline Preoperative membranous urethral length $(\mathrm{mm})$ & $\geq 15 \mathrm{~mm}$ & $<15 \mathrm{~mm}$ \\
\hline Membranous urethral length-removal rate & $\otimes 10 \%$ & $\geq 10 \%$ \\
\hline Shape of vesicourethral anastomosis & Triangle & Square \\
\hline
\end{tabular}

\section{Figures}




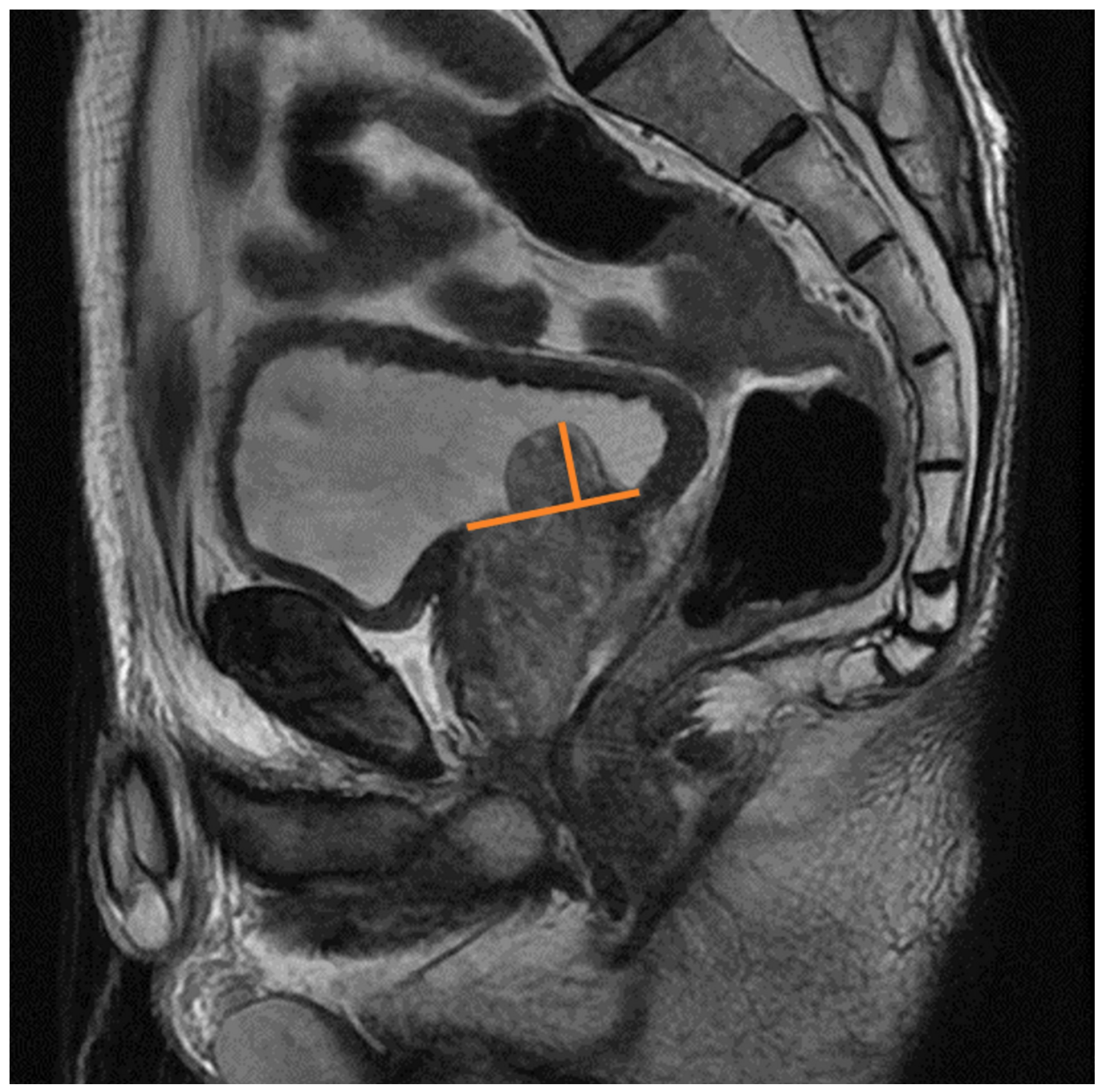

\section{Figure 1}

The vertical distance from the tip of the protruding prostate to the base of the urinary bladder on the midsagittal image defined as intravesical prostatic protrusion length (IPPL). 


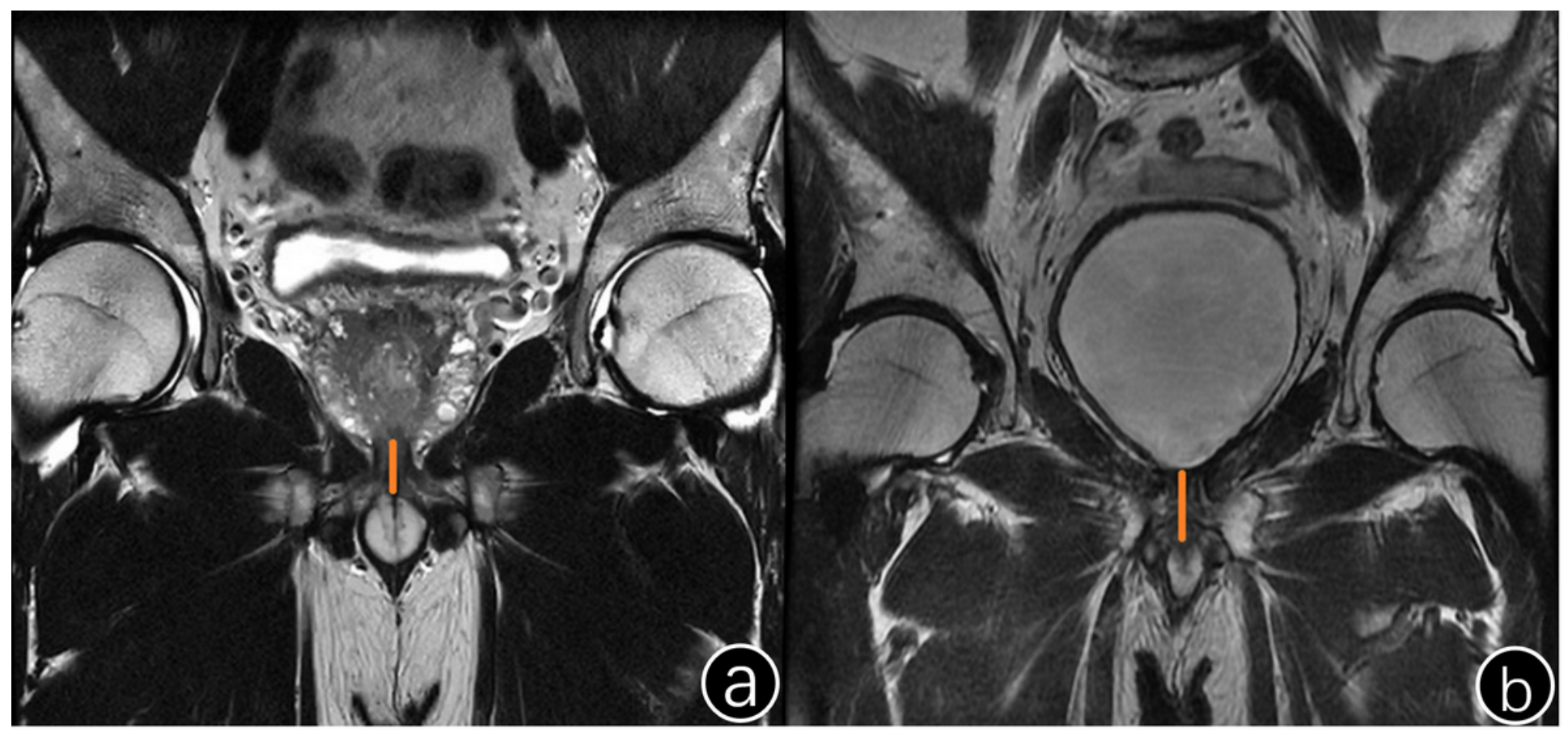

\section{Figure 2}

The distance from the apex of prostate / bladder neck to the urethra at the level of the penile bulb on the coronal image defined as preoperative MUL (a) / postoperative MUL (b). MUL membranous urethral length.

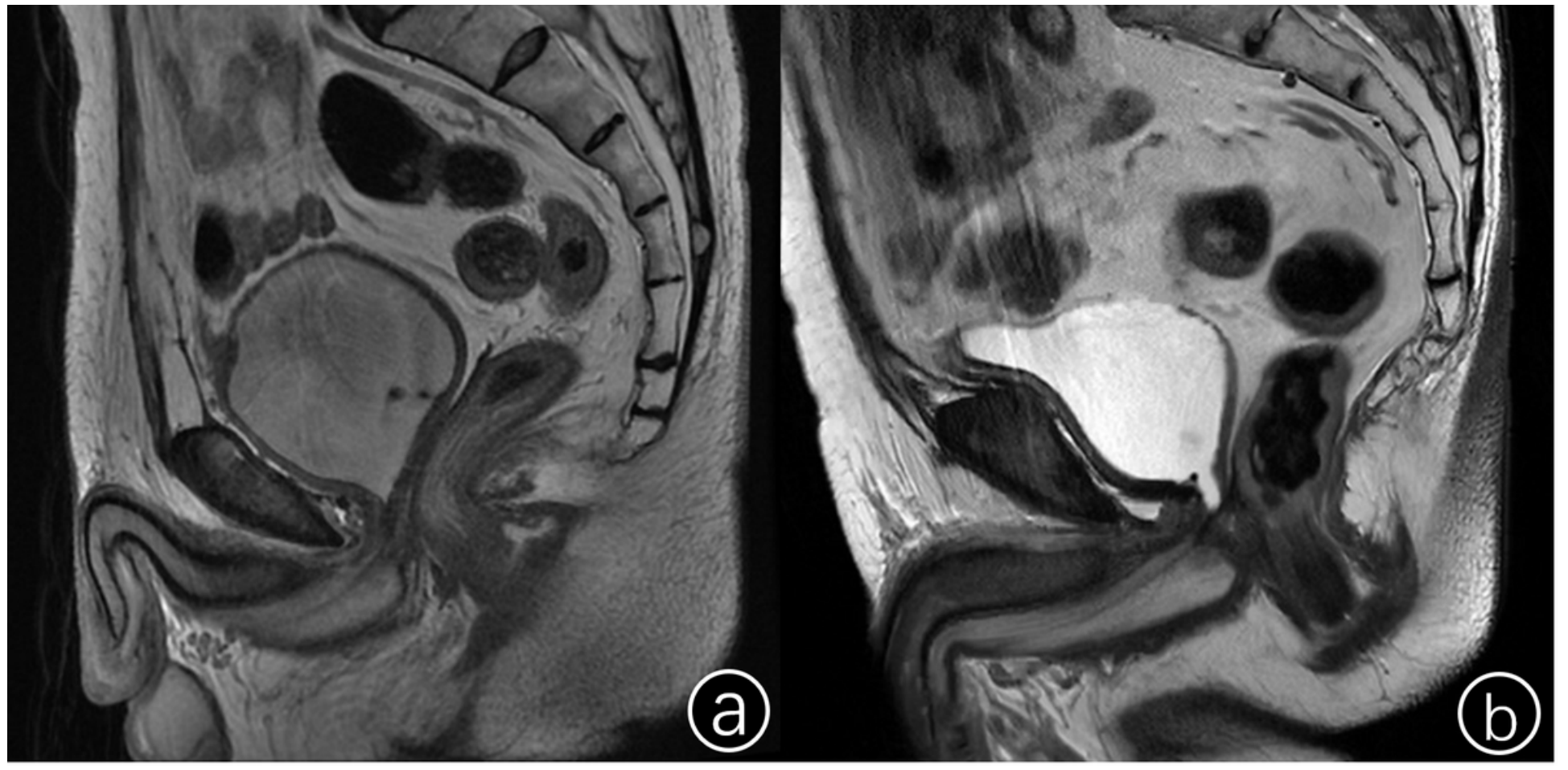

Figure 3 
The shape of vesicourethral anastomosis (VUA) on the midsagittal image of postoperative magnetic resonance imaging: Triangle VUA (a) and square VUA (b).

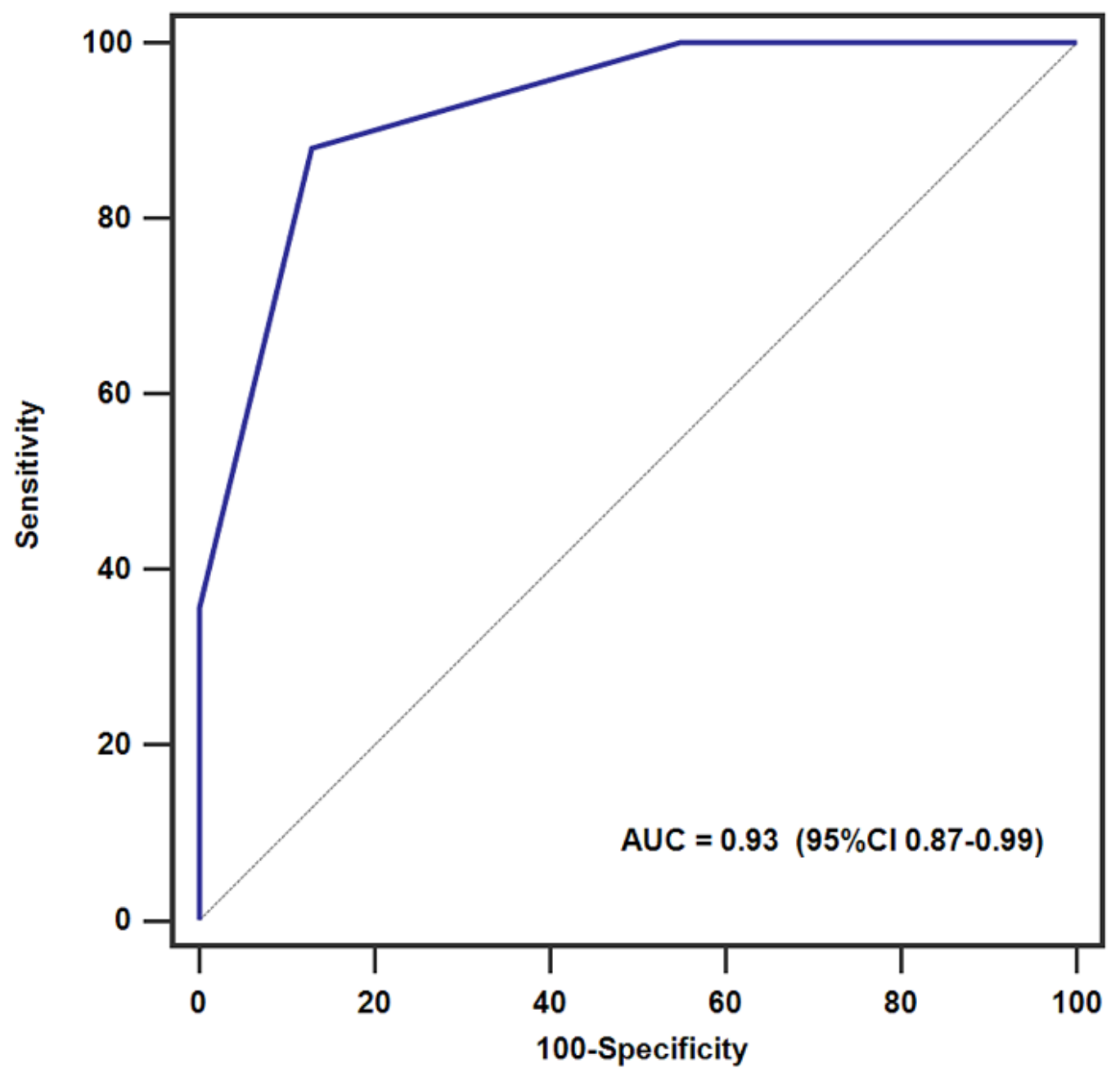

Figure 4

ROC curve of Post-Prostatectomy Incontinence Score predicting early continence recovery after laparoscopic radical prostatectomy. ROC receiver operating characteristic, AUC area under the ROC curve.

\section{Supplementary Files}

This is a list of supplementary files associated with this preprint. Click to download.

- SupplementalFig1.tif 